\title{
Des délégués syndicaux sous-payés : une situation de discrimination stratégique?
}

Une analyse économétrique à partir de l'enquête REPONSE de 2010

Are Union Representatives Underpaid in France Due to Strategic

Discrimination? An Empirical Assessment Based on the 2010 REPONSE Survey

Jérôme Bourdieu et Thomas Breda

\section{OpenEdition}

\section{Journals}

Édition électronique

URL : http://journals.openedition.org/travailemploi/6888

DOI : 10.4000/travailemploi.6888

ISSN : 1775-416X

Éditeur

DARES - Ministère du Travail

Édition imprimée

Date de publication : 1 janvier 2016

Pagination : 31-58

ISSN : 0224-4365

\section{Référence électronique}

Jérôme Bourdieu et Thomas Breda, «Des délégués syndicaux sous-payés : une situation de discrimination stratégique? », Travail et Emploi [En ligne], 145 | janvier-mars 2016, mis en ligne le 01 janvier 2018, consulté le 20 avril 2019. URL : http://journals.openedition.org/travailemploi/6888 ; DOI : 10.4000/travailemploi.6888 


\title{
Des délégués syndicaux sous-payés : une situation de discrimination stratégique?
}

\section{Une analyse économétrique à partir de l'enquête REPONSE de 2010*}

\author{
Jérôme Bourdieu ${ }^{* *}$, Thomas Breda ${ }^{* * *}$
}

\begin{abstract}
Les cas de discrimination à l'encontre des syndicalistes s'accumulent. En témoignent la multiplication des procédures judiciaires et les condamnations dont ont fait l'objet de nombreuses grandes entreprises françaises. Ce constat qualitatif a été conforté par les résultats statistiques obtenus à partir de l'enquête Relations professionnelles et négociations d'entreprise (REPONSE) de 2004 qui font apparaître qu'à niveaux de diplôme et âges égaux, les délégués syndicaux sont payés environ $10 \%$ de moins que leurs collègues, ce que confirme, on le montre ici, la version 2010 de l'enquête. L'appartenance à un syndicat ne suffit pas à expliquer ces moins bons salaires. Ce sont en effet les représentants syndiqués, les plus actifs dans la défense des intérêts de leurs collègues, qui sont les plus pénalisés. Il est, en pratique, difficile d'établir que ces salariés ont des salaires inférieurs pour d'autres raisons que leurs responsabilités syndicales. Les pénalités salariales pour les syndiqués sans mandat restent en effet assez faibles - de l'ordre de $4 \%$-tandis qu'inversement, ce sont dans les entreprises où il y a des conflits que les délégués syndicaux sont le plus pénalisés.
\end{abstract}

$\mathrm{D}$ ans une situation économique de chômage durable et de croissance faible, la perspective de perdre son emploi effraie, et la défense des intérêts des travailleurs peut alors devenir de plus en plus difficile. Dans ce contexte, les indices qualitatifs suggérant l'existence d'une discrimination de grande ampleur à l'encontre des syndicalistes

\footnotetext{
* Les auteurs ont bénéficié d'un financement de la Direction de l'animation de la recherche, des études et des statistiques (Dares) pour cette recherche.

** Paris School of Economics (PSE) ; Institut national de recherche agronomique (Inra) ; École des hautes études en sciences sociales (EHESS) ; jerome.bourdieu@ens.fr.

*** PSE ; Centre national de la recherche scientifique (CNRS) ; thomas.breda@ens.fr.
} 
s'accumulent ${ }^{1}$. Depuis les années 2000, de nombreuses grandes entreprises françaises ont ainsi dû indemniser des dizaines de représentants à la suite de procédures judiciaires pour discrimination syndicale (Semaine sociale Lamy, 2004).

Ces cas particuliers pourraient avoir un caractère exceptionnel ou atypique ; les témoignages individuels ou les ressentis personnels pourraient ne pas correspondre à une situation partagée par le plus grand nombre. Les résultats obtenus à partir de l'enquête Relations professionnelles et négociations d'entreprise (REPONSE) de 2004 produite par la Direction de l' animation de la recherche, des études et des statistiques (Dares) rendent compte au contraire du caractère général de ce constat et font apparaître l'existence d'un écart de rémunération de l'ordre de $10 \%$ entre les délégués syndicaux et leurs collègues, une fois prises en compte leurs caractéristiques observables, telles que le sexe, l'âge, le niveau de diplôme, etc. (BREDA, 2014). Dans le même temps, la pénalité observée pour les salariés qui sont syndiqués sans être délégués syndicaux est plus faible et n'est pas significative statistiquement.

Ces résultats suggèrent que les salariés ne sont pas pénalisés de la même façon selon les modalités de leur engagement dans la défense de leurs intérêts collectifs. Pour conforter et approfondir ce constat, nous utilisons les données de la dernière version de l'enquête REPONSE qui permet d'analyser plus finement ces modalités d'engagement. Elle inclut en effet une nouvelle question qui offre la possibilité de produire pour la première fois en 2010 des comparaisons de salaire directes entre les représentants du personnel syndiqués et non syndiqués, et leurs collègues. L'objectif est d'examiner ici si les pénalités subies sont imputables au fait d'être syndiqué et/ou de participer à des instances de représentation dans l'entreprise. Une analyse plus poussée de la manière dont la défense des intérêts des salariés est organisée dans l'entreprise est alors possible, qui prend en compte à la fois le type d'instance de négociation et l'appartenance syndicale.

Grâce à une méthode d'estimation indirecte, ces analyses font apparaître la situation propre des délégués syndicaux : comme nous allons le voir, ils subissent les pénalités salariales les plus fortes ; les autres syndiqués, qu'ils soient représentants ou non, sont moins pénalisés ; et les représentants non syndiqués ne le sont pas du tout. L'analyse des promotions, passées ou à venir, et des catégories socioprofessionnelles des différents groupes de salariés considérés indique de plus que les écarts de salaire observés résultent de taux de promotions différenciés. Par ailleurs, ces écarts de salaires ne concernent pas toutes les entreprises. Par exemple, lorsqu'une entreprise négocie spécifiquement sur le droit syndical, ou lorsque les négociations annuelles obligatoires n'ont pas lieu (alors qu'elles le devraient), les représentants syndiqués et les délégués syndicaux ne subissent plus aucune pénalité salariale. La présentation de ces résultats statistiques sera l'objet de notre première partie.

1. Voir par exemple les nombreux témoignages recueillis dans le rapport annuel de l'Observatoire des répressions et discriminations syndicales. 
Ensuite, après avoir explicité notamment ce qui caractérise la position de délégué syndical dans le processus de négociation, nous proposons une interprétation théorique des pénalités salariales mises en évidence économétriquement : elles seraient le résultat d'une discrimination "stratégique », ayant pour but de préserver les intérêts des employeurs pour qui la négociation, salariale en particulier, peut être coûteuse. Les autres interprétations possibles (discrimination « par goût », anti-sélection, discrimination statistique, phénomène d'autocensure, cf. p. 46 et suivantes) sont également considérées et discutées, notamment au regard de ce qui fait la spécificité de l'engagement syndical. Les données disponibles ne permettant pas de conclure quant aux mécanismes causaux à l'œuvre, la discussion a pour principal objectif de clarifier les différents concepts de discrimination ou d'anti-sélection, ainsi que leurs limites lorsqu'on les applique aux représentants du personnel. En effet, ces derniers dédient une partie de leur temps de travail à leur activité de représentation : ils ne font donc jamais exactement le même travail que leurs collègues non-représentants, de telle sorte qu'il est formellement impossible de démontrer qu'à travail égal, ils n'obtiennent pas un salaire égal.

\section{Écarts de salaire et de taux de promotion entre les représentants du personnel, les syndiqués, et les autres salariés}

Les données de l'enquête REPONSE de 2010 (voir encadré 1) permettent d'identifier les représentants du personnel et de mesurer leurs salaires. Elle fournit donc une mesure directe de la pénalité salariale qu'ils sont susceptibles de subir en exerçant une

\section{ENCADRÉ 1 \\ L'enquête REPONSE appariée aux $D A D S^{1}$}

Le travail empirique est réalisé à partir des trois volets des enquêtes REPONSE de 2004 et $2010^{2}$.

Produite par la Dares, l'enquête REPONSE est l'une des principales sources statistiques sur les relations professionnelles en France. Elle est menée tous les six ans auprès d'un échantillon qui, en 2010 comportait environ 4000 établissements de 10 salariés ou plus du secteur marchand non agricole ${ }^{3}$. Dans chacun de ces établissements, un représentant

1. Déclarations annuelles de données sociales.

2. Les enquêtes portent en fait sur les années 2004-2005 et 2010-2011. Mais elles sont appariées à des données de salaire issues des DADS pour les années 2003 et 2009. Pour simplifier, nous parlons d'estimations pour les années 2004 et 2010, mais ces légers anachronismes doivent être gardés en tête.

3. Lors des éditions antérieures, l'enquête couvrait les établissements des entreprises de 50 salariés ou plus en 1992 et les établissements de 20 salariés ou plus en 1998 et 2004, à chaque fois dans le secteur marchand non agricole. 
de la direction est interrogé lors d'un long entretien en face-à-face. Il répond à un grand nombre de questions portant notamment sur l'organisation du travail, les technologies utilisées et les relations professionnelles. Les réponses des représentants de la direction constituent le volet «employeurs » de l'enquête. Lorsqu'il y a un ou des représentants du personnel dans l'établissement, on demande à l'employeur de donner le nom d'un représentant du syndicat ou de la liste majoritaire dans son établissement. Ces représentants sont ensuite interrogés. On dispose ainsi en 2010 d'un échantillon de 2433 représentants qui forment le volet « représentants du personnel » de l'enquête. Un volet « salariés » est finalement constitué par tirage au sort de 10 salariés par établissement qui ont reçu un questionnaire par courrier (dans chaque établissement, tous les salariés ayant plus d'un an d'ancienneté ont la même probabilité d'être tirés). Du fait d'un taux de réponse de l'ordre de $30 \%$, usuel pour les enquêtes par courrier, nous disposons in fine d'un peu moins de 3 salariés en moyenne par établissement dans l'échantillon final. Une fois les volets « employeurs » et « salariés » appariés, nous obtenons ainsi, en 2010, un échantillon de 11334 salariés dans 4023 établissements.

Les volets «salariés » des enquêtes REPONSE de 2004 et de 2010 ayant été au préalable appariés par la Dares avec les $D A D S$, nous avons connaissance du salaire horaire net de chaque salarié présent dans l'échantillon final au 31 décembre 2009 pour l'enquête de 2010, et au 31 décembre 2003 pour l'enquête de 2004. Les DADS sont en outre utilisées comme source alternative pour quelques autres variables, telles que la taille des établissements ou le salaire moyen en leur sein.

fonction officielle de représentation des intérêts des salariés. Cette mesure peut être conçue comme une vérification des résultats obtenus pour les délégués syndicaux sur les données de l'enquête REPONSE de 2004 (BREDA, 2014), qui montraient par une méthode d'inférence indirecte l'existence d'une telle pénalité : les délégués syndicaux étaient payés en moyenne $10 \%$ de moins que les collègues qu'ils représentaient à caractéristiques observables égales (diplôme, âge, ancienneté, etc.). Ces pénalités salariales se renforçaient avec leur ancienneté, la discrimination qu'ils déclaraient ressentir, et leur appartenance à un syndicat plus contestataire tel que la Confédération générale du travail (CGT).

\section{Salaires des représentants syndiqués et non syndiqués, et des syndiqués non-représentants}

Le tableau 1 présente en détail les estimations de l'écart relatif de salaire entre les représentants, syndiqués et non syndiqués, et les salariés qui ne sont ni représentants ni syndiqués (pris comme catégorie de référence ${ }^{2}$ ). Les écarts de salaire moyens

\footnotetext{
2. Les estimations sont réalisées sur un échantillon de salariés en contrat à durée indéterminée (CDI), à temps complet, hors apprentis et stagiaires, hors emplois secondaires, avec des heures déclarées dans l'année comprises entre 900 et 2 200, travaillant dans des établissements de 10 salariés et plus du secteur marchand non agricole. Elles sont pondérées pour garantir la représentativité des salariés au sein de l'échantillon. Ces choix et leur incidence sur les résultats sont discutés en annexe.
} 
TABLEAU 1 - Écarts de salaires entre les représentants du personnel, les syndiqués et leurs collègues en 2009

Variable dépendante : $\log$ du salaire horaire (DADS 2009)

\begin{tabular}{|c|c|c|c|c|c|c|c|}
\hline & (1) & (2) & (3) & (4) & (5) & (6) & (7) \\
\hline $\begin{array}{l}\text { Syndiqué non } \\
\text { représentant }\end{array}$ & $\begin{array}{c}0,014 \\
(0,014)\end{array}$ & $\begin{array}{c}-0,038 * * * \\
(0,013)\end{array}$ & $\begin{array}{c}-0,044 * * * \\
(0,013)\end{array}$ & $\begin{array}{l}-0,002 \\
(0,011)\end{array}$ & $\begin{array}{c}-0,033 * * * \\
(0,012)\end{array}$ & $\begin{array}{c}-0,037 * * * \\
(0,012)\end{array}$ & $\begin{array}{l}-0,008 \\
(0,010)\end{array}$ \\
\hline $\begin{array}{l}\text { Représentant } \\
\text { syndiqué }\end{array}$ & $\begin{array}{l}-0,003 \\
(0,018)\end{array}$ & $\begin{array}{c}-0,035^{* * *} \\
(0,016)\end{array}$ & $\begin{array}{c}-0,046 * * * \\
(0,016)\end{array}$ & $\begin{array}{l}-0,001 \\
(0,013)\end{array}$ & $\begin{array}{c}-0,034 * * \\
(0,014)\end{array}$ & $\begin{array}{c}-0,049 * * * \\
(0,014)\end{array}$ & $\begin{array}{l}-0,011 \\
(0,011)\end{array}$ \\
\hline $\begin{array}{l}\text { Représentant } \\
\text { non syndiqué }\end{array}$ & $\begin{array}{l}-0,012 \\
(0,021)\end{array}$ & $\begin{array}{l}-0,005 \\
(0,019)\end{array}$ & $\begin{array}{l}-0,017 \\
(0,019)\end{array}$ & $\begin{array}{l}-0,001 \\
(0,016)\end{array}$ & $\begin{array}{c}0,005 \\
(0,016)\end{array}$ & $\begin{array}{l}-0,008 \\
(0,016)\end{array}$ & $\begin{array}{l}-0,001 \\
(0,013)\end{array}$ \\
\hline Observations & 12,318 & 7,695 & 7,606 & 7,606 & 12,189 & 12,046 & 12,046 \\
\hline$R^{2}$ & 0,000 & 0,490 & 0,503 & 0,667 & 0,794 & 0,801 & 0,871 \\
\hline $\begin{array}{l}\text { Contrôles } \\
\text { salariés }\end{array}$ & Non & Mincer & $\begin{array}{c}\text { Mincer }+ \\
\text { ancienneté }\end{array}$ & Détaillés & Mincer & $\begin{array}{l}\text { Mincer }+ \\
\text { ancienneté }\end{array}$ & Détaillés \\
\hline $\begin{array}{l}\text { Contrôles } \\
\text { établissements }\end{array}$ & Non & Oui & Oui & Oui & Effets fixes & Effets fixes & Effets fixes \\
\hline Pondéré & Oui & Oui & Oui & Oui & Oui & Oui & Oui \\
\hline
\end{tabular}

Note : toutes les régressions sont pondérées au niveau des salariés à l'aide des poids fournis par la Dares avec l'enquête.

Les contrôles individuels notés « Mincer » sont le diplôme ( 9 catégories), l'âge (en années), l'âge au carré et le sexe. Les contrôles individuels « Détaillés » incluent en plus l'ancienneté, l'ancienneté au carré, la catégorie socioprofessionnelle (4 groupes) et le nombre d'heures travaillées. Les contrôles établissements « Standards » sont la taille (5 groupes), le secteur (16 groupes), la région (10 groupes), l'âge (5 groupes) et une indicatrice pour la présence d'un délégué syndical. Écarts types entre parenthèses. $* * * \mathrm{p}<0.01, * * \mathrm{p}<0.05, * \mathrm{p}<0.1$.

Lecture : en l'absence de variables de contrôle (colonne 1), le logarithme du salaire d'un syndiqué non représentant est supérieur en moyenne de 0,014 au logarithme du salaire d'un salarié qui n'est ni représentant ni syndiqué (catégorie de référence).

Champ : salariés des établissements de 10 salariés et plus du secteur marchand non agricole en CDI à temps complet, hors apprentis et stagiaires, hors emplois secondaires, avec heures déclarées annuelles entre 900 et 2200.

Source : enquête REPONSE, 2010, volet « salariés », apparié aux DADS, et volet « employeurs ».

(sans variables de contrôle) entre ces différentes catégories de salariés sont faibles (inférieurs à 1,5\%) et non significatifs (colonne 1). Lorsqu'on évalue une équation de salaire linéaire qui inclut des variables de contrôle pour le niveau d'instruction, le genre, l'âge et l'âge au carré des salariés ainsi que les caractéristiques de base de leur établissement de travail (secteur d'activité, taille, âge et présence d'un délégué syndical $^{3}$ ), on trouve une pénalité salariale de 3,5\% pour les représentants syndiqués par rapport aux salariés qui ne sont ni représentants ni syndiqués. Les représentants non syndiqués ne subissent pas, eux, de pénalité salariale, tandis que les syndiqués non-représentants subissent également une pénalité d'environ 3,5\% par rapport aux non syndiqués non-représentants (colonne 2).

Ce sont principalement les contrôles pour l'âge des salariés et la taille des établissements qui expliquent la variation des écarts de salaire estimés entre les colonnes 1 et 2 : les représentants du personnel sont plus âgés et présents dans des établissements plus grands que l'ensemble des salariés (voir tableau 2), et l'âge comme la taille des

3. La méthode de régression linéaire utilisée ici permet d'estimer la relation linéaire qui relie le salaire à un certain nombre d'autres variables qui peuvent être d'intérêt (ici le fait d'être syndiqué et/ou représentant) ou de contrôle. Les écarts de salaire ainsi estimés entre syndiqués et/ou représentants sont corrigés des différences observables entre ces salariés en termes des variables de contrôle introduites dans la régression. 
TABLEAU 2 - Proportion de syndiqués et de représentants du personnel selon les caractéristiques économiques des établissements et sociodémographiques des salariés

\begin{tabular}{|c|c|c|c|c|c|c|}
\hline & $\begin{array}{l}\text { Représentant } \\
\text { du personnel }\end{array}$ & $\begin{array}{l}\text { Représentant } \\
\text { non syndiqué }\end{array}$ & $\begin{array}{c}\text { Représentant } \\
\text { syndiqué }\end{array}$ & Syndiqué & $\begin{array}{c}\text { Ancien } \\
\text { syndiqué }\end{array}$ & $\begin{array}{l}\text { Proportion de } \\
\text { représentants } \\
\text { parmi les } \\
\text { syndiqués }\end{array}$ \\
\hline Ensemble & 6,4 & 2,8 & 3,6 & 11,0 & 8,9 & 32,9 \\
\hline \multicolumn{7}{|l|}{ Sexe } \\
\hline Hommes & 6,9 & 2,9 & 4,0 & 11,8 & 10,1 & 34,1 \\
\hline Femmes & 5,7 & 2,7 & 3,0 & 9,8 & 7,2 & 31,0 \\
\hline \multicolumn{7}{|c|}{ Catégorie socioprofessionnelle } \\
\hline Ouvrier & 6,7 & 2,6 & 4,1 & 12,8 & 10,0 & 32,4 \\
\hline Employé & 6,3 & 2,9 & 3,3 & 9,6 & 7,6 & 34,4 \\
\hline $\begin{array}{l}\text { Profession } \\
\text { intermédiaire }\end{array}$ & 7,3 & 3,1 & 4,3 & 12,6 & 10,3 & 33,9 \\
\hline Cadre & 4,9 & 2,7 & 2,2 & 7,4 & 6,9 & 30,2 \\
\hline \multicolumn{7}{|l|}{ Diplôme } \\
\hline Aucun diplôme & 5,4 & 2,2 & 3,1 & 13,1 & 10,6 & 23,7 \\
\hline $\begin{array}{l}\text { Niveau inférieur } \\
\text { au baccalauréat }\end{array}$ & 6,9 & 2,6 & 4,3 & 12,3 & 12,0 & 35,3 \\
\hline Baccalauréat & 6,4 & 3,0 & 3,4 & 12,1 & 8,2 & 28,3 \\
\hline $\begin{array}{l}\text { Niveau supérieur } \\
\text { au baccalauréat }\end{array}$ & 6,3 & 3,1 & 3,2 & 8,7 & 5,7 & 36,8 \\
\hline \multicolumn{7}{|l|}{ Temps de travail } \\
\hline Temps complet & 6,8 & 2,9 & 4,0 & 11,3 & 9,0 & 34,9 \\
\hline Temps partiel & 4,0 & 2,2 & 1,8 & 9,2 & 8,2 & 19,8 \\
\hline \multicolumn{7}{|l|}{ Secteur } \\
\hline Industrie & 6,8 & 3,0 & 3,8 & 10,4 & 8,2 & 36,8 \\
\hline Construction & 6,7 & 2,4 & 4,3 & 15,6 & 14,8 & 27,7 \\
\hline Commerce & 7,4 & 3,6 & 3,9 & 8,8 & 5,8 & 44,3 \\
\hline Services & 6,5 & 2,9 & 3,5 & 10,8 & 8,8 & 32,9 \\
\hline \multicolumn{7}{|c|}{ Taille d'établissement } \\
\hline 10-19 salariés & 5,0 & 3,3 & 1,7 & 5,0 & 7,3 & 34,0 \\
\hline 20-49 salariés & 6,3 & 3,7 & 2,5 & 7,8 & 7,9 & 32,6 \\
\hline 50-99 salariés & 9,0 & 4,9 & 4,2 & 9,9 & 9,8 & 42,6 \\
\hline 100-199 salariés & 8,1 & 3,7 & 4,5 & 13,3 & 8,6 & 33,6 \\
\hline 200-999 salariés & 6,5 & 1,0 & 5,5 & 15,2 & 9,0 & 36,2 \\
\hline$>1000$ salariés & 3,6 & 0,2 & 3,4 & 14,7 & 12,2 & 23,0 \\
\hline
\end{tabular}

Note : toutes les statistiques sont pondérées au niveau des salariés à l'aide des poids fournis par la Dares avec l'enquête. Champ : salariés des établissements de 10 salariés et plus du secteur marchand non agricole.

Source : enquête REPONSE, 2010, volet « salariés », apparié aux DADS, et volet « employeurs ». 
établissements sont associés à des salaires plus élevés. Un contrôle additionnel pour l'ancienneté (linéaire et au carré) des salariés annule la prime salariale pour les syndiqués non-représentants et fait passer la pénalité salariale pour les représentants syndiqués à 4,5\% (colonne 3 du tableau 1) : les syndiqués et les représentants ont une ancienneté dans l'établissement plus importante que leurs collègues et des salaires relativement meilleurs de ce fait.

L'ajout de contrôles supplémentaires pour le nombre d'heures travaillées et surtout la catégorie socioprofessionnelle des salariés (répartis en quatre groupes de qualification : ouvriers, employés, professions intermédiaires et cadres) réduit à zéro les écarts de salaires entre représentants et/ou syndiqués et leurs collègues (colonne 4) : si on compare les représentants aux autres salariés à catégories socioprofessionnelles égales, la pénalité disparaît. Cela ne signifie pas pour autant, on y reviendra, qu'il n'existe pas de pénalité mais que ces pénalités salariales ne peuvent être pensées en dehors d'autres paramètres de choix des employeurs, tels que les promotions par exemple, qui peuvent également être utilisées à des fins discriminatoires.

Une dernière série de spécifications vise à prendre en compte l'existence de différences éventuelles entre établissements en matière de politique salariale en introduisant des effets fixes. Les colonnes 5 à 7 du tableau 1 reproduisent les colonnes 2 à 4 , avec cette fois des effets fixes par établissement plutôt que des contrôles dont la valeur varie par établissement. Conceptuellement, cela revient à faire des comparaisons fines entre les représentants et/ou les syndiqués, et leurs collègues qui ne sont ni l'un ni l'autre, établissement par établissement, de telle sorte que les écarts de salaires entre ces différentes catégories de salariés ne puissent pas s'expliquer par des différences de salaire moyen entre, par exemple, les établissements dans lesquels il y a des représentants, et ceux où il n'y en a pas. Les résultats dans ces spécifications avec effets fixes sont semblables aux résultats sans effets fixes.

$\mathrm{Au}$ total, nous retenons que l'écart de salaire entre les salariés syndiqués (représentants ou non), et leurs collègues non syndiqués (représentants ou non) est, en 2010, d'environ $-3,5 \%$ lorsque l'on considère des salariés de même sexe, même expérience, même niveau d'instruction, et travaillant dans le même établissement. Ces résultats suggèrent que c'est l'appartenance à un syndicat plutôt que le fait d'occuper une fonction de représentant qui génère des pénalités salariales.

\section{Comme en 2004, des délégués syndicaux moins bien payés en 2010}

La pénalité subie par les représentants syndiqués en 2010 est nettement plus faible que celle subie par les délégués syndicaux en 2004 (telle qu'estimée par T. BREDA en 2014). De nombreuses raisons peuvent expliquer cet écart. D'une part, des différences de méthodes sont intervenues dans la mesure de la pénalité salariale : cette dernière était indirecte et moins précise en 2004 ; le champ de l'enquête a changé pour inclure les entreprises de dix à vingt salariés; et la variable d'intérêt n'est pas la même, puisque l'on est passé des délégués syndicaux aux représentants syndiqués. D’autre 
part, l'écart de pénalité salariale entre 2004 et 2010 pourrait refléter des changements réels intervenus au cours de la période. Les tests que nous avons effectués indiquent que le changement de variable d'intérêt est la principale explication de la différence de résultats (voir BOURDIEU, BREDA, 2015, pour une analyse détaillée).

Si la distinction entre un représentant syndiqué et un délégué syndical peut apparaître minime à un non-spécialiste des relations professionnelles, elle ne l'est en fait ni au regard de l'attribution des délégués, ni au regard de leur nombre au sein du contingent plus vaste des représentants syndiqués. Les estimations menées à partir de l'enquête REPONSE indiquent qu'il y a en France dans les établissements de plus de dix salariés du secteur marchand non agricole environ 100000 délégués syndicaux et 300000 représentants syndiqués (voir tableau 3). Cela signifie que seul un représentant syndiqué sur trois est en fait délégué syndical. Les autres représentants syndiqués peuvent être, par exemple, délégués du personnel, responsables de section syndicale ou encore représentants syndiqués au sein du comité d'entreprise, de la délégation unique du personnel ou du comité d'hygiène, de sécurité et des conditions de travail (CHSCT). La différence principale entre les délégués syndicaux et les autres représentants tient à leurs attributions : le délégué syndical a la responsabilité de négocier les accords d'entreprise avec l'employeur, notamment lors des négociations annuelles obligatoires portant sur les salaires et les conditions de travail, tandis que les autres représentants (syndiqués) ont essentiellement un rôle de consultation et de vecteur de communication entre salariés et employeurs. Engagés dans la négociation qui, si elle fonctionne efficacement, impliquera un partage de la valeur ajoutée moins avantageux pour les employeurs, les délégués syndicaux forment un groupe à part. Et c'est au sein de ce groupe que les pénalités salariales sont les plus importantes.

Pour estimer la pénalité salariale spécifique des délégués syndicaux, il est nécessaire d'utiliser, sur les données de 2010, une méthode indirecte analogue à celle

\section{TABLEAU 3 - Répartition des salariés selon qu'ils sont syndiqués et/ou représentants}

En unité et \%

\begin{tabular}{|c|c|c|c|c|c|c|}
\hline \multirow[b]{2}{*}{ Non syndiqués, non représentants } & \multicolumn{2}{|c|}{ Échantillon complet } & \multicolumn{2}{|c|}{ Échantillon d'analyse } & \multicolumn{2}{|c|}{$\begin{array}{c}\text { Champ de l'enquête } \\
\text { (en milliers de salariés } \\
\text { pour la colonne } \\
\text { de gauche) }\end{array}$} \\
\hline & 15702 & 86,1 & 10832 & 85,3 & 7921 & 86,2 \\
\hline Représentants non syndiqués & 543 & 3,0 & 405 & 3,2 & 256 & 2,8 \\
\hline Syndiqués, non représentants & 1316 & 7,2 & 908 & 7,2 & 678 & 7,4 \\
\hline Représentants syndiqués & 672 & 3,7 & 548 & 4,3 & 332 & 3,6 \\
\hline dont Délégués Syndicaux & $\approx 124$ & $\approx 0,7$ & $\approx 93$ & $\approx 0,7$ & $\approx 93$ & $\approx 1,0$ \\
\hline Total & 18233 & 100,0 & 12693 & 100,0 & 9188 & 100,0 \\
\hline
\end{tabular}

Note : toutes les statistiques sont obtenues en utilisant les pondérations salariées fournies par la Dares. Pour le détail de l'estimation du nombre de délégués syndicaux, voir BouRDIEu et BREDA, 2015.

Champ : salariés des établissements de 10 salariés et plus du secteur marchand non agricole en CDI à temps complet, hors apprentis et stagiaires, hors emplois secondaires, avec heures déclarées annuelles entre 900 et 200.

Source : enquête REPONSE, 2010, volet « salariés », apparié aux DADS, et volet « employeurs ». 
mobilisée sur les données de 2004 (voir encadré 2). Ces derniers ne sont en effet pas directement identifiables dans le volet « salariés » de l'enquête REPONSE de 2010.

En adoptant la méthode utilisée en 2004 qui identifie la probabilité d'être délégué via leur proportion parmi les syndiqués, on trouve une pénalité salariale moyenne de l'ordre de $6 \%$ pour les délégués lorsqu'on contrôle pour le niveau d'instruction, le sexe et l'expérience des salariés, et les caractéristiques principales de leur établissement. Cette pénalité passe à $5 \%$ lorsqu'on inclut des effets fixes par établissement. Ces écarts de salaire estimés ne sont pas statistiquement significatifs du fait de l'imprécision inhérente à la méthode utilisée, mais ils vont dans le sens d'une plus forte pénalité pour les délégués syndicaux.

\section{ENCADRÉ 2}

\section{Une méthode et trois stratégies d'identification indirecte des délégués syndicaux en 2010}

On peut estimer la probabilité qu'un salarié se déclarant syndiqué soit délégué syndical en exploitant les réponses des représentants de la direction sur le nombre de délégués syndicaux et le pourcentage de syndiqués dans leur établissement. En multipliant le pourcentage de syndiqués déclaré par l'employeur par la taille de chaque établissement (connue via les $D A D S$ ), on obtient le nombre de syndiqués. En divisant ensuite le nombre de délégués syndicaux par le nombre de syndiqués, on calcule la proportion de délégués parmi les syndiqués au niveau de chaque établissement. Les salariés interrogés dans l'enquête étant tirés au hasard, cette proportion correspond à la probabilité qu'un salarié d'un établissement donné soit délégué syndical. Si le salarié ne s'est pas déclaré syndiqué, sa probabilité d'être délégué syndical est égale à zéro car les délégués doivent nécessairement être syndiqués. Si le salarié est syndiqué, alors sa probabilité d'être délégué est égale à la proportion de délégués parmi les syndiqués dans son établissement de travail. La probabilité ainsi calculée qu'un salarié soit délégué est une information suffisante pour estimer l'écart de salaire moyen entre les délégués syndicaux et leurs collègues (sous l'hypothèse que cet écart ne varie pas avec cette probabilité, voir BREDA, 2014).

C'est cette méthode « probabiliste » qui avait été utilisée sur les données de 2004. En exploitant la nouvelle question de l'enquête de 2010 permettant d'identifier les salariés qui sont représentants du personnel, il est possible de la raffiner afin d'estimer plus précisément les salaires des délégués syndicaux. L'idée est d'exploiter cette fois-ci les déclarations des employeurs sur le nombre de représentants et de représentants syndiqués pour identifier les délégués syndicaux, non pas parmi l'ensemble des syndiqués, mais parmi l'ensemble plus restreint des représentants, voire parmi l'ensemble encore plus restreint des représentants syndiqués. La pénalité pour les délégués est alors obtenue en croisant le fait qu'un salarié se déclare représentant du personnel (respectivement représentant du personnel syndiqué) avec la probabilité qu'un représentant du personnel (respectivement représentant du personnel syndiqué) soit délégué syndical. Cette méthode nécessite notamment d'émettre des hypothèses sur le cumul des mandats parmi les représentants syndiqués (voir pour les détails BOURDIEU, BREDA, 2015). 
Les trois stratégies d'estimation reprenant la méthode de mesure probabiliste qui permet d'identifier indirectement des représentants en 2010 (encadré 2), confirment largement ce constat. Une fois contrôlée des différences d'expérience et de diplôme entre les salariés et des différences de taille et de secteur d'activité de leur établissement, la pénalité pour les délégués est estimée à environ $5 \%$ lorsqu'ils sont identifiés parmi l'ensemble des représentants, et à $12 \%$ lorsqu'ils sont identifiés de manière plus certaine parmi le groupe plus restreint des représentants syndiqués. Ces pénalités sont plus fortes, de l'ordre de 10 à $15 \%$ dans les spécifications qui nous semblent les plus adaptées et qui incluent des effets fixes par établissement ${ }^{4}$.

Chacune des trois méthodes d'identification probabiliste des délégués syndicaux aboutit à des résultats peu précis au sens statistique du fait de l'identification indirecte des délégués syndicaux. Lorsqu'elle est faite à partir des représentants du personnel, cette identification probabiliste est cependant améliorée par rapport à 2004, en raison de la proportion plus élevée des délégués syndicaux parmi les représentants que parmi les syndiqués. Par ailleurs, les trois mesures du salaire que nous proposons convergent dans le sens de pénalités plus élevées à l'égard des délégués syndicaux. Compte tenu du faisceau d'indices disponibles, nous retenons un ordre de grandeur de $10 \%$ pour la pénalité salariale des délégués syndicaux, dont l'estimation reste cependant imprécise.

En résumé, l'analyse des écarts de salaires liés à l'appartenance syndicale révèle un double phénomène : les syndiqués subissent une perte de salaire significative par rapport à leurs collègues non syndiqués ; cette perte est aggravée par le fait d'avoir certaines responsabilités officielles dans la représentation des salariés. Et on ne peut imputer entièrement les écarts de salaire au seul fait d'être représentant, puisqu'on ne les observe pas pour les représentants qui ne sont pas syndiqués.

\section{Des écarts de salaire expliqués par des taux de promotion différenciés}

Les pénalités salariales, que ce soit pour l'ensemble des salariés syndiqués, les représentants syndiqués ou encore plus précisément les délégués syndicaux, disparaissent lorsque la qualification exacte est incluse comme variable de contrôle (voir tableau 1, colonnes 4 et 7). Ce résultat montre que ces pénalités s'expliquent par le fait que, même à âge, sexe et niveau de diplôme identiques, les représentants et les syndiqués n'occupent pas les mêmes postes que leurs collègues. Il suggère également que les pénalités salariales peuvent découler de taux différenciés de promotion, puisque la qualification présente dépend de promotions antérieures.

C'est ce que confirme le tableau 4 , qui montre qu'à âge, sexe et niveau d'instruction égaux, les syndiqués (qu'ils soient ou non représentants) ont, au moins, $5 \%$ de chances de moins de déclarer avoir obtenu une promotion au cours des trois dernières années que les salariés qui ne sont ni représentants ni syndiqués (colonnes 1 et 2). Les déclarations

4. L'ensemble de ces résultats est détaillé dans Bourdieu, BredA (2014) : tableaux 5A, 5B, 5C, et A3, A4, A5. 
Des délégués syndicaux sous-payés : une situation de discrimination stratégique?

TABLEAU 4 - Différences de (chances de) promotion, d'augmentation de salaire et de risque de licenciement entre les représentants, les syndiqués et leurs collègues

\begin{tabular}{|c|c|c|c|c|c|c|}
\hline & \multicolumn{2}{|c|}{ Promu les 3 dernières années } & \multicolumn{2}{|c|}{$\begin{array}{l}\text { Chances de promotion ou } \\
\text { d'augmentation de salaire au } \\
\text { cours des } 12 \text { prochains mois }\end{array}$} & \multicolumn{2}{|c|}{$\begin{array}{c}\text { Risque de licenciement } \\
\text { au cours } \\
\text { des } 12 \text { prochains mois }\end{array}$} \\
\hline & (1) & (2) & (3) & (4) & (5) & (6) \\
\hline $\begin{array}{l}\text { Syndiqué non } \\
\text { représentant }\end{array}$ & $\begin{array}{c}-0,049^{* *} \\
(0,020)\end{array}$ & $\begin{array}{c}-0,080 * * * \\
(0,021)\end{array}$ & $\begin{array}{c}-0,134 * * * \\
(0,050)\end{array}$ & $\begin{array}{c}-0,238 * * * \\
(0,052)\end{array}$ & $\begin{array}{c}0,257 * * * * \\
(0,052)\end{array}$ & $\begin{array}{c}0,191 * * * \\
(0,050)\end{array}$ \\
\hline $\begin{array}{l}\text { Représentant } \\
\text { syndiqué }\end{array}$ & $\begin{array}{c}-0,058^{* *} \\
(0,024)\end{array}$ & $\begin{array}{c}-0,074 * * * \\
(0,025)\end{array}$ & $\begin{array}{c}-0,214 * * * \\
(0,059)\end{array}$ & $\begin{array}{c}-0,236 * * * \\
(0,060)\end{array}$ & $\begin{array}{c}0,269 * * * \\
(0,061)\end{array}$ & $\begin{array}{c}0,170 * * * \\
(0,056)\end{array}$ \\
\hline $\begin{array}{l}\text { Représentant } \\
\text { non syndiqué }\end{array}$ & $\begin{array}{c}0,084 * * * \\
(0,030)\end{array}$ & $\begin{array}{c}0,080 * * * \\
(0,029)\end{array}$ & $\begin{array}{c}0,228 * * * \\
(0,072)\end{array}$ & $\begin{array}{c}0,222 * * * \\
(0,072)\end{array}$ & $\begin{array}{l}-0,107 \\
(0,071)\end{array}$ & $\begin{array}{l}-0,061 \\
(0,066)\end{array}$ \\
\hline Observations & 7,369 & 11,705 & 6,175 & 9,800 & 5,927 & 9,397 \\
\hline$R^{2}$ & 0,062 & 0,517 & 0,082 & 0,591 & 0,049 & 0,651 \\
\hline $\begin{array}{l}\text { Contrôles } \\
\text { salariés }\end{array}$ & Mincer & Mincer & Mincer & Mincer & Mincer & Mincer \\
\hline $\begin{array}{l}\text { Contrôles } \\
\text { établissements }\end{array}$ & Oui & Effets fixes & Oui & Effets fixes & Oui & Effets fixes \\
\hline Pondéré & Oui & Oui & Oui & Oui & Oui & Oui \\
\hline
\end{tabular}

Note : les contrôles sont définis dans le tableau 4. Résultats obtenus via des modèles de probabilité linéaire (OLS). Les variables de chance de promotion ou d'augmentation et de risque de licenciement sont standardisées pour avoir un écart type de 1.

Lecture : un représentant syndiqué a 5,8\% de chances de moins de déclarer avoir reçu une promotion dans les trois années précédant l'enquête qu'un salarié non représentants non syndiqué (colonne 1).

Champ : salariés des établissements de 10 salariés et plus du secteur marchand non agricole en CDI à temps complet, hors apprentis et stagiaires, hors emplois secondaires, avec heures déclarées annuelles entre 900 et 2200.

Source : enquête REPONSE, 2010, volets « salariés » et « employeurs ».

des salariés vont dans le même sens que les estimations obtenues concernant leurs salaires puisqu'elles ne permettent pas non plus de distinguer les salariés syndiqués qui sont représentants des autres salariés syndiqués : le fait d'être représentant n'accroît pas la tendance, propre aux salariés syndiqués, à être moins souvent promus.

En réalité, le phénomène est plus complexe encore : on observe, d'une part, que les délégués syndicaux sont, eux, effectivement encore moins souvent promus que les salariés syndiqués (les résultats statistiques ne sont pas fournis ici) mais, en même temps, que les représentants non syndiqués sont nettement plus souvent promus que les salariés ordinaires. Plus précisément, sur l'ensemble de l'échantillon, la proportion de salariés qui déclarent avoir eu une promotion dans les trois années précédant l'enquête est de $25 \%$ : cela signifie que les taux de promotion déclarés varient presque du simple au double entre les représentants syndiqués et leurs collègues représentants non syndiqués (de $17 \%$ à $33 \%$ dans les spécifications avec effets fixes de la colonne $2^{5}$ ).

5. Ces fortes différences en termes de promotions passées se retrouvent dans les déclarations des salariés quant à leurs chances de promotion ou d'augmentation (colonnes 3 et 4). Pour établir ces résultats, on ne contrôle que pour les différences entre salariés selon des caractéristiques individuelles qui ne sont pas manipulables par l'employeur : l'âge, le sexe et le niveau d'instruction. 
Ce contraste très fort entre représentants syndiqués et non syndiqués se retrouve lorsque l'on examine non pas les promotions passées mais les chances déclarées de promotion ou d'augmentation dans l'année à venir (tableau 4, colonnes 3 et 4). Il est enfin également visible en ce qui concerne le risque perçu de licenciement dans l'année à venir (colonnes 5 et 6). On constate en particulier que la protection contre le licenciement dont bénéficient les représentants syndiqués ne les empêche pas d'estimer avoir davantage de chances d'être licenciés que les autres salariés.

\section{Les pénalités salariales ne sont pas systématiques}

L'enquête REPONSE permet de savoir si des négociations relatives au droit syndical et au droit à l'expression des salariés ont eu lieu au niveau des établissements et des entreprises qu'elle couvre, et si ces négociations ont abouti à un accord. Si, en théorie, les négociations identifiées dans REPONSE peuvent ne pas concerner du tout la carrière des représentants syndicaux, en pratique, selon les praticiens du droit social, les accords sur le droit syndical mettent souvent en place une gestion des carrières des représentants du personnel. C'est également ce que confirment les monographies réalisées par Vincent-Arnaud CHAPPE et ses co-auteures (2015). On peut donc faire l'hypothèse que les négociations sur le droit syndical et l'expression des salariés identifiées dans l'enquête REPONSE fournissent un bon indicateur de l'existence de négociations portant au moins en partie sur la carrière des représentants syndicaux.

Entre 2008 et 2010, environ $30 \%$ des établissements (45\% de ceux ayant des délégués syndicaux) ont ainsi négocié sur le droit syndical et/ou le droit d'expression des salariés au niveau de l'établissement et/ou de l'entreprise, et environ la moitié de ces négociations a abouti à un accord (voir tableau 5). Lorsqu'on se restreint aux établissements appartenant aux entreprises de plus de 300 salariés pour lesquels une obligation de négocier sur le droit syndical a été introduite par la loi du 20 août 2008 (tableau 5), les taux de négociation sur le droit syndical augmentent encore (ils doublent pratiquement), restant toutefois largement inférieurs à $100 \%$, ce qui indique que les obligations légales ne sont pas toujours respectées ${ }^{6}$. Les comparaisons entre 2004 et 2010 (voir tableau 5) suggèrent cependant que la loi a été tout de même mise en œuvre dans un certain nombre d'entreprises : les négociations et signatures d'accords concernant le droit syndicat ou le droit d'expression des salariés ont augmenté sur la période, notamment dans les établissements d'entreprises de plus de 300 salariés.

\footnotetext{
6. Il est possible qu'elles soient respectées au niveau de l'entreprise mais que le représentant de la direction au niveau de l'établissement l'ignore. Une telle ignorance montrerait alors que les accords sur le droit syndical négociés au niveau de l'entreprise ne sont pas appliqués dans tous les établissements. On peine à croire cependant que ce cas de figure puisse prévaloir dans les $40 \%$ d'établissements disposant de délégués syndicaux et appartenant à une entreprise de plus de 300 salariés pour lesquels les représentants de direction déclarent qu'il n'y a pas eu de négociation sur le droit syndical. Il y a donc certainement une part importante des établissements qui n'appliquent pas (toutes) les dispositions de la loi du 20 août 2008.
} 


\section{TABLEAU 5 - Prévalence du recours au droit et de la négociation concernant le droit syndical} entre 2008 et 2010 et entre 2002 et 2004

En \% d'établissements

Place du droit syndical et/ou de l'expression des salariés dans les relations sociales de l'établissement (déclarations des dirigeants interrogés)

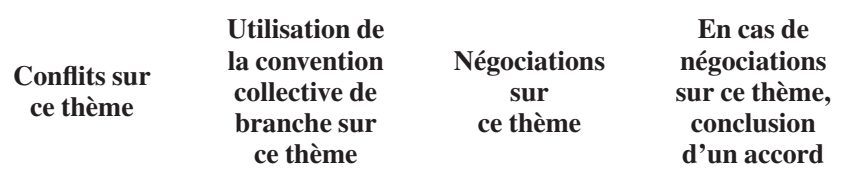

\section{Entre 2008 et 2010}

Tous établissements

Établissements dont l'entreprise a plus de 300 salariés

Établissements avec représentant du personnel interrogé

Établissements avec représentant du personnel dont l'entreprise a plus de 300 salariés

Établissements avec délégué syndical

Établissements avec délégué syndical dont l'entreprise a plus de 300 salariés
1,9

71,6

30,2

46,8

58,5

61,9

2,2

76,3

34,4

46,5

57,7

56,8

3,2

79,4

44,8

53,2

57,3

59,5

Entre 2002 et 2004

Tous établissements

2,3

69,5

32,7

40,1

Établissements dont l'entreprise a plus de 300 salariés

Établissements avec représentant du personnel interrogé

69,5

Établissements avec représentant du personnel dont l'entreprise a plus de 300 salariés

Établissements avec délégué syndical

Établissements avec délégué syndical dont l'entreprise a plus de 300 salariés

Note : statistiques établies à partir de questions posées aux représentants de direction et portant sur l'existence de conflits, de négociations ou d'accords au cours des trois dernières années précédant l'enquête (période 2008-2010 pour REPONSE, 2010, et période 2002-2004 pour REPONSE, 2004).

Lecture : la proportion d'établissements du champ ayant eu entre 2008 et 2010 des négociations sur le droit syndical ou l'expression des salariés est de 30,2\%. Cette proportion est de 58,5\% parmi les établissements de plus de 300 salariés, de 34,4 \% parmi ceux ayant un représentant interrogé, et de 44,8\% parmi ceux disposant d'au moins un délégué syndical.

Champ : établissements de 10 salariés et plus du secteur marchand non agricole.

Source : enquêtes REPONSE, 2004 et 2010, volets « employeurs ». 
TABLEAU 6 - Écarts de salaires entre les représentants, les syndiqués et leurs collègues en 2009 suivant l'existence de négociations sur le droit syndical

\begin{tabular}{|c|c|c|c|c|}
\hline & \multicolumn{4}{|c|}{ Effet pour les établissements ayant des délégués syndicaux } \\
\hline & \multicolumn{2}{|c|}{$\begin{array}{c}\text { Pas de négociation sur le droit } \\
\text { syndical }\end{array}$} & \multicolumn{2}{|c|}{ Négociation sur le droit syndical } \\
\hline & (1) & (2) & (3) & (4) \\
\hline Syndiqué non représentant & $\begin{array}{c}-0,074 * * * \\
(0,018)\end{array}$ & $\begin{array}{c}-0,094 * * * \\
(0,021)\end{array}$ & $\begin{array}{l}-0,010 \\
(0,023)\end{array}$ & $\begin{array}{c}0,016 \\
(0,027)\end{array}$ \\
\hline Représentant syndiqué & $\begin{array}{c}-0,065 * * * \\
(0,022)\end{array}$ & $\begin{array}{c}-0,052^{* *} \\
(0,024)\end{array}$ & $\begin{array}{l}-0,027 \\
(0,028)\end{array}$ & $\begin{array}{l}-0,002 \\
(0,031)\end{array}$ \\
\hline Représentant non syndiqué & $\begin{array}{l}-0,010 \\
(0,040)\end{array}$ & $\begin{array}{l}-0,005 \\
(0,041)\end{array}$ & $\begin{array}{l}-0,000 \\
(0,056)\end{array}$ & $\begin{array}{c}0,015 \\
(0,062)\end{array}$ \\
\hline Observations & 2,650 & 2,661 & 1,824 & 1,825 \\
\hline$R^{2}$ & 0,499 & 0,767 & 0,539 & 0,769 \\
\hline Contrôles salariés & Mincer & Mincer & Mincer & Mincer \\
\hline Contrôles établissements & Oui & Effets fixes & Oui & Effets fixes \\
\hline Pondéré & Oui & Oui & Oui & Oui \\
\hline Établissements avec délégué syndical & Oui & Oui & Oui & Oui \\
\hline Négociations sur le droit syndical & Non & Non & Oui & Oui \\
\hline
\end{tabular}

Effet pour les délégués syndicaux (ensemble des établissements)

Méthode 1 :

Délégué syndical

$\begin{array}{cccc}-0,106^{* *} & -0,148^{* * *} & 0,016 & -0,031 \\ (0,050) & (0,054) & (0,058) & (0,069)\end{array}$

Représentant non délégué syndical

$-0,002$

0,021

$-0,047$

0,010

$(0,021)$

$(0,023)$

$(0,030)$

$(0,035)$

Méthode 2 :

Délégué syndical

$\begin{array}{rrrr}-0,095 * & -0,049 & -0,016 & -0,075 \\ (0,052) & (0,057) & (0,070) & (0,085)\end{array}$

Syndiqué non délégué

$$
-0,059 * * *
$$$$
-0,066^{* * * *}
$$

$(0,019)$

$(0,022)$

$(0,024)$

$(0,029)$

\section{Méthode 3 :}

Délégué syndical

$\begin{array}{cccc}-0,210 * * * & -0,239 * * * & -0,034 & -0,079 \\ (0,078) & (0,086) & (0,081) & (0,094) \\ 0,023 & 0,062 & 0,009 & 0,082 \\ (0,059) & (0,065) & (0,070) & (0,078)\end{array}$

Note : voir les notes du tableau 1, l'encadré 2 et BouRdiEu et BREDA (2015) pour les détails concernant les méthodes 1, 2 et 3 aboutissant aux estimations «probabilistes » portant sur les seuls délégués syndicaux.

Lecture : dans les établissements n'ayant pas négocié sur le droit syndical, les syndiqués non représentants sont payés en moyenne environ $7,4 \%$ de moins que leurs collègues qui ne sont ni représentants ni syndiqués (catégorie de référence), à diplôme, sexe, âge et caractéristiques d'établissement comparables, colonne 1.

Champ : salariés des établissements de 10 salariés et plus du secteur marchand non agricole en CDI à temps complet, hors apprentis et stagiaires, hors emplois secondaires, avec heures déclarées annuelles entre 900 et 2200.

Source : enquête REPONSE, 2010, volet « salariés », apparié aux DADS, et volet « employeurs ». 
Le tableau 6 montre que la négociation d'accords de droit syndical est associée à une disparition des pénalités salariales subies par les représentants et les délégués syndicaux. Plus précisément, on voit (par estimation directe) que les syndiqués comme les représentants syndiqués subissent une pénalité salariale en 2009 (contrairement aux représentants non syndiqués) uniquement dans les établissements où il n'y a pas eu de négociations sur les droits syndicaux entre 2008 et 2010. Les pénalités salariales pour les représentants syndiqués ou les délégués syndicaux ne seraient donc pas immuables et la négociation sur ces questions peut sans doute les diminuer. Il n'est cependant pas possible de conclure à un effet causal de la négociation sur le droit syndical. Il se pourrait par exemple que les établissements dans lesquels les représentants sont le mieux payés soient plus prompts à négocier sur le droit syndical, auquel cas nos résultats refléteraient simplement cette sélection. Il semble cependant que la menace de contentieux pour discrimination syndicale (ChapPe, 2015 ; SEMAine Sociale Lamy, 2004) soit, au moins dans certains cas, à l'origine des négociations sur le droit syndical. Ces dernières ne correspondent donc pas toujours à une simple mise en conformité légale de la part des seules entreprises qui rémunèrent leurs représentants au même niveau que les autres salariés.

\section{Comment comprendre la pénalisation salariale subie par les délégués syndicaux ?}

Pour le sens commun, une pénalité salariale à expérience et niveau de diplôme identiques exprime une forme de discrimination. Cependant, l'économiste aura tendance, en l'absence d'autres éléments probants, à attribuer un écart de salaire à des différences inobservées de compétence ou de productivité. De la même façon, en droit, la preuve juridique de la discrimination salariale serait pour ainsi dire systématiquement réfutée " au bénéfice du doute » sauf dans les cas exceptionnels où le salarié, sur qui repose la charge de la preuve, est en mesure de démontrer qu'il n'est pas moins productif. Cependant, des aménagements importants du cadre légal concernant la charge de la preuve tendent à faire maintenant reposer sur l'employeur la justification d'écarts de salaires liés à des écarts de productivité (CHAPPE, 2013).

La discrimination salariale est définie tant en droit qu'en économie comme la violation d'un principe : principe d'équité pour l'économie - « à productivité égale, salaire égal »- ; principe d'égalité en droit - « à travail égal, salaire égal ». Parce que le travail de deux salariés est en réalité toujours différent, et parce que la productivité (marginale) individuelle n'est presque jamais totalement observable (notamment lorsque le processus de production est collectif), ces principes sont pratiquement invérifiables, ce qui rend toute preuve avérée de discrimination salariale quasiment impossible, tant par l'économiste, le salarié demandant réparation ou encore l'employeur accusé de pratiques discriminatoires. 
Si elles compliquent l'établissement de preuves statistiques définitives, ces difficultés n'interdisent pas pour autant de discuter les différents mécanismes capables de générer des écarts de salaire, qu'ils relèvent effectivement d'une forme de discrimination, ou d'une sélection de salariés considérés comme moins compétents. Nous tentons de distinguer cinq mécanismes complémentaires générateurs d'écarts de salaire et de taux de promotion. Les trois premiers mécanismes sont la discrimination par goût (BECKER, 1971), l'anti-sélection (qui correspond à l'idée selon laquelle les représentants du personnel seraient moins productifs) et la discrimination statistique (ARROW, 1973 ; PHELPS, 1972). Ces mécanismes font partie de la grille de lecture classiquement utilisée en économie pour expliquer des écarts observables entre groupes sociaux. À ces explications « classiques », nous proposons d'ajouter une forme de discrimination atypique, qui s'explique par des motifs stratégiques et qui est spécifique à la représentation collective des intérêts des salariés. C'est cette explication qui nous semble la plus probante, bien que nous ne soyons pas en mesure de rejeter les autres et qu'elles peuvent, en certains cas, être complémentaires. Enfin, nous discutons l'existence possible d'un comportement d'autocensure de la part des représentants du personnel, qui renonceraient à mettre en avant leur situation individuelle pour mieux se consacrer à la défense des autres salariés.

\section{La discrimination « par goût »}

La discrimination par goût à l'encontre d'un groupe social traduit simplement le fait que l'employeur a une préférence pour les individus ne faisant pas partie de ce groupe. Une hostilité de principe à l'égard des syndicats et des syndicalistes se traduirait ainsi par une pénalisation salariale des salariés syndiqués. Elle ne peut, au moins pour des raisons légales, servir de justification aux choix d'embauche, de rémunération ou de licenciement d'un salarié mais de nombreux témoignages en attestent l'existence?

L'idée d'une discrimination par goût à l'encontre des syndicalistes est toutefois difficile à dissocier du rôle économique et social qu'ils jouent dans l'entreprise, sauf à considérer que les syndicalistes aient des caractéristiques propres et indépendantes de ce rôle, qui les rendraient antipathiques aux yeux du patron. En effet, l'aversion que peut ressentir un employeur à l'égard des syndicats n'est probablement pas totalement indépendante du fait que les syndicats, incarnés par les délégués syndicaux, représentent des intérêts opposés aux siens. En ce sens, la discrimination par goût rejoint la discrimination stratégique que nous discutons plus loin, même si la première renvoie surtout à une opposition globale entre syndicats et employeurs alors que la seconde relie localement, au niveau de chaque établissement, une éventuelle discrimination à l'encontre de certains représentants liée à leur rôle particulier au cours des négociations.

7. Michelin est un cas exemplaire : voir Mousli M. (2011), «Un patron paternaliste et antisyndical », Alternatives économiques, $\mathrm{n}^{\mathrm{o}} 305$, septembre 2011, p. 78. 


\section{L'anti-sélection}

Plutôt qu'une aversion des employeurs, les pénalités salariales des salariés engagés dans le syndicalisme pourraient refléter leur plus faible productivité. Tel peut être l'avis de certaines directions d'entreprise qui interprètent l'engagement syndical comme une manière de fuir le travail productif et de «tirer au flanc». Les salariés syndiqués seraient moins productifs que les autres, soit parce que ne se syndiqueraient et, plus encore, ne feraient une carrière syndicale que des individus particulièrement peu engagés dans le travail ; soit parce que le fait de devenir syndicaliste détournerait les individus de leur activité (plus directement) productive au-delà des seules heures de délégations. Les taux de promotion plus faibles des syndiqués s'expliqueraient alors par une plus faible productivité dont auraient connaissance les employeurs.

Ces points sont presque impossibles à évaluer empiriquement : aucune base de données n'est assez riche pour permettre de produire une mesure exacte de la productivité des salariés, pour autant que celle-ci soit effectivement mesurable. Cependant, plusieurs remarques peuvent être faites concernant cette interprétation, qui est aussi une justification parfois avancée par les employeurs. D'abord, les pénalités salariales augmentent avec l'ancienneté des salariés dans l'établissement : elles sont ainsi inexistantes pour les salariés ayant peu d'ancienneté, tant dans l'enquête de 2004 que dans celle de $2010^{8}$. Les salariés qui deviennent syndiqués ou représentants du personnel ne sont donc pas initialement moins bien rémunérés, ce qui suggère (sans pour autant le démontrer) qu'ils n'étaient pas déjà moins productifs lorsqu'ils se sont engagés. Autrement dit, les représentants et les syndiqués ne semblent pas systématiquement sélectionnés parmi les salariés moins payés et moins productifs avant leur engagement dans le syndicalisme.

Si c'est un différentiel de productivité entre les représentants ou les syndiqués et leurs collègues qui explique les écarts de salaire, il est plus vraisemblable que ce différentiel apparaisse après la prise de mandat : c'est lorsqu'ils s'engagent dans le syndicalisme ou en tant que représentant que les salariés commenceraient à moins s'investir dans leur travail. Cette éventualité invite à un commentaire, qui concerne spécifiquement les représentants du personnel.

Ces derniers, du fait des heures de délégation, ont « statutairement » des activités qui ne sont plus exactement comparables à celles de leurs collègues sur l'ensemble de leur temps de travail. Les dispositions légales particulières qui s'appliquent aux représentants interdisent tout écart de salaire s'expliquant par l'existence de ces délégations horaires dont le coût est à la charge de l'employeur. Les délégations horaires affectent cependant l'organisation du travail, et pourraient notamment expliquer les taux de

\footnotetext{
8. Voir BREDA (2014) et BouRdieU, BREDA (2015). La difficulté tient ici à ce que seule l'ancienneté dans l'établissement est connue lors de l'analyse des salaires. Mais des analyses complémentaires à partir du volet « représentant du personnel » de l'enquête REPONSE révèlent que c'est bien l'ancienneté comme représentant dans l'établissement qui explique le sentiment de discrimination ressenti par les représentants, et non leur ancienneté comme salarié dans l'établissement (BREDA, 2014 ; BourdiEU, BredA, 2015).
} 
promotion différentiés : si un employeur doit promouvoir un salarié à un poste qui requiert une charge de travail importante, il est possible qu'il préfère celui qui n'a pas de délégation horaire et qui peut par conséquent s'investir à temps plein dans son nouveau travail ; dans ce numéro l'article de V.-A. CHAPPE et ses co-auteures témoigne de telles situations. Si l'on souhaite reprendre les catégorisations classiques, doit-on dans un tel cas conclure qu'il y a discrimination ou qu'il y a anti-sélection?

D'un point de vue économique, la différence d'activité de travail effective suffit formellement à rendre caduque la possibilité de parler de discrimination dans ce cas. Adopter un tel point de vue en pratique serait un moyen de légitimer systématiquement les pénalités salariales subies par les représentants du personnel syndiqués et cela, quels que soient les motifs réels ayant entraîné ces pénalités. La réponse juridique actuelle se distingue de cette approche et opte, au contraire, pour l'interprétation la plus favorable aux salariés, à savoir l'existence d'une discrimination, même s'il est impossible de vérifier dans ce cas précis la condition «à travail égal, salaire égal ». Les nombreux procès gagnés pour discrimination syndicale en témoignent.

Une voie alternative, plus juste si l'on s'en tient au strict point de vue économique, serait de valoriser pour elle-même l'activité de représentation en mettant en œuvre des mécanismes de reconnaissance des compétences acquises par les représentants du personnel. Dans ce sens, la loi du 17 août 2015 rend par exemple obligatoire l'évaluation des compétences acquises au cours du mandat par des instances de certification externes ou directement par les responsables des ressources humaines de leur entreprise. Les dispositifs de certification externe, qui existaient déjà avant 2015, semblent cependant insuffisants pour déboucher sur de réelles évolutions de carrière. Ils ne concernent de plus presque que des permanents syndicaux ou des grands mandatés, laissant démunie la majorité des représentants ne disposant que d'une petite délégation horaire (CHAPPE et al., 2015). L'évaluation des compétences acquises par le représentant syndical directement par l'entreprise ou par son service de ressources humaines se heurte quant à elle à un problème fondamental : dès lors qu'il y a conflit entre les intérêts des salariés et des employeurs (ce qui est généralement le cas quand il s'agit de partager les bénéfices), les représentants se retrouvent face au risque de « déplaire » à leur employeur. On peut légitimement se demander comment une évaluation des compétences syndicales par les employeurs peut fonctionner en pratique : n'est-ce pas là institutionnaliser une forme de dialogue social qui correspond plus aux attentes des employeurs qu'à celles des salariés ?

\section{La discrimination statistique}

Une forme de discrimination directement liée à l' anti-sélection légitime les moins bonnes carrières des syndicalistes au nom de la croyance, fondée ou non fondée, qu'ils sont moins compétents. L'argument n'est valable que dans un environnement où la productivité et le travail effectué sont imparfaitement observables. Dans ce cas, l'employeur pourra avoir recours à des informations ne concernant pas directement 
la productivité ou les compétences, mais qui permettraient de les révéler. Ce ne serait alors pas la moins bonne productivité des syndiqués qui expliquerait directement leurs moins bons salaires, mais la croyance de l'employeur que cette productivité est moins bonne. Un tel argument est en général évoqué pour les discriminations à l'embauche plutôt que pour les discriminations au travail qui s'inscrivent en principe dans des interactions de long terme telles que le travail accompli par les uns et les autres est observable.

Un phénomène similaire à l'anti-sélection dynamique décrite ci-dessus est cependant susceptible d'être à l'œuvre : l'employeur pourrait penser que la syndicalisation ou la participation aux instances de représentation du personnel ne sont pas l'indication de moins bonnes compétences initiales, mais plutôt d'un désinvestissement au travail. Considérant, à tort ou à raison, que les syndicalistes ne sont plus prêts à faire d'efforts, les employeurs seraient alors moins enclins à les promouvoir et à les rétribuer. Comme le montrent les travaux sur la discrimination statistique (par exemple ceux de LundBerg, STARTZ 1983 ; CoATE, LOURY 1993 ; LOURY 2002), les croyances sont parfois auto-réalisatrices : sachant par avance que les employeurs considèrent leur engagement comme une forme de désinvestissement au travail, les syndicalistes n'auraient alors plus d'intérêt à s'impliquer dans un travail qui ne sera vraisemblablement pas évalué à sa juste valeur. L'absence d'effort ainsi rationalisée des syndicalistes devrait alors se coupler à un autre phénomène, également rationnel, de sélection à l'entrée des seuls salariés prêts à renoncer à leur carrière professionnelle. $\mathrm{Si}$ ces situations sont décrites ici de façon un peu caricaturale, il ne faut pas négliger l'existence de ces cercles vicieux qui, à partir de visions négatives sur le dialogue social et ses acteurs de la part des employeurs, aboutissent à un dialogue social effectivement conflictuel et incarné par des représentants bien particuliers : ceux qui sont prêts à renoncer à leur carrière.

Les témoignages issus d'enquêtes de terrain amènent cependant à nuancer l'existence de tels phénomènes : certains des salariés syndiqués, notamment ceux qui exercent un mandat de représentant dans l'entreprise, sont parfois décriés, y compris par d'autres salariés, et qualifiés de «planqués » qui tirent parti de leur décharge syndicale et de leur statut de salarié protégé pour ne rien faire ; mais ils sont aussi souvent considérés comme des individus particulièrement actifs et entreprenants, parfois surinvestis dans l'entreprise, dans leurs activités militantes comme dans leur travail, ne serait-ce que pour ne pas se mettre en faute vis-à-vis d'un employeur prêt à les renvoyer au moindre écart dûment constaté. En témoignent par exemple les statistiques sur les heures de délégation : les représentants syndiqués, et particulièrement les délégués syndicaux, disposent plus fréquemment que les représentants non syndiqués d'un crédit d'heures officiel, et celui-ci est en moyenne supérieur. Pour autant, ils déclarent très souvent le dépasser et consacrer davantage de temps à leur activité de représentant (PAK, PIGNONI, 2014). Cela est d'autant plus le cas lorsqu'ils sont affiliés à la CGT (huit fois plus de chances de dépasser le crédit d'heures que les représentants non syndiqués, à caractéristiques de l'établissement et de l'entreprise équivalentes), à l'Unsa (Union 
nationale des syndicats autonomes), Solidaires ou à un syndicat autonome (six fois plus de chances). L'histoire du licenciement de Nordine, syndicaliste Sud à GRDF, et de la lutte sur très longue durée menée par son syndicat et ses membres pour obtenir la reconnaissance du caractère discriminatoire de cette décision et sa réintégration est l'un des nombreux exemples documentés montrant que les délégués syndicaux s'investissent pour fournir un travail de qualité même si, bien évidemment, en cas de conflit, leur employeur ne le reconnaît pas (BARNIER et al., 2011).

\section{La discrimination stratégique}

L'hostilité à l'égard des syndicalistes pourrait être, plus spécifiquement, la conséquence de la position revendicatrice que traduit l'appartenance à un syndicat. D'un point de vue théorique, au-delà donc d'une aversion de principe à l'égard de l'activité syndicale, ou d'une compensation économique de leur moindre productivité, réelle ou supposée, les salaires moindres des représentants syndiqués tiendraient alors au fait qu'ils expriment des intérêts divergents, émanant en partie d'organisations syndicales extérieures à l'entreprise. En ce cas, l'employeur serait tenté de pénaliser les syndicalistes indépendamment de la qualité de leur travail en tant que salariés et ce, d'autant plus que leur investissement syndical est susceptible de menacer les intérêts patronaux au cours des négociations ${ }^{9}$. En ce sens, effectivement, les pénalités subies par le salarié syndiqué, parce qu'elles sont indépendantes de son travail comme salarié, relèvent de la discrimination au sens juridique.

La discrimination exercée par l'employeur pourrait ainsi résulter d'un comportement stratégique qui tient non pas au salarié lui-même mais aux revendications qu'il porte et risque de porter avec succès. Son but serait alors de dissuader les salariés de devenir délégués syndicaux, de participer aux négociations d'entreprise et de décourager les mobilisations et les revendications salariales.

L'efficacité d'une telle pratique ne tient donc pas seulement à l'effet qu'elle produit sur les délégués syndicaux eux-mêmes mais également au signal qu'elle transmet à l'ensemble des salariés afin de les dissuader de soutenir l'action syndicale. Ces effets dissuasifs indirects peuvent être importants. Un sondage TNS-SOFRES montre par exemple que plus d'un tiers des salariés mentionne la peur des représailles comme une raison à la faible syndicalisation en France. L'absence de délégués syndicaux dans environ deux tiers des établissements de plus de dix salariés (calculs des auteurs), au sein desquels la loi prévoit pourtant leur présence, est un autre indice que beaucoup de salariés hésitent à s'engager dans le syndicalisme. Si la stratégie consistant à discriminer les délégués syndicaux les plus actifs ne garantit pas leur affaiblissement

\footnotetext{
9. Comme le montre de manière systématique et frappante le numéro spécial que la revue Agone consacre à ce sujet, la discrimination des syndicats ne résulte pas (seulement) d'une sorte d'effet moyen cumulé statistiquement mais au contraire de politiques patronales ciblées sur certains individus et elle s'exprime bien autrement que sur le seul terrain des salaires. Il existe en réalité de véritables « techniques de domestication des conflits du travail » pour reprendre le titre de l'article de Baptiste GIRAUD (2013) dans ce même volume.
} 
immédiat, elle a de bonnes chances d'affaiblir la portée de l'action collective à moyen terme, d'une part, parce que beaucoup de salariés hésiteront à soutenir directement l'action des syndicats concernés et, d'autre part, parce que d'autres salariés iront même jusqu'à se désolidariser de ces syndicats du fait du signal très négatif envoyé par l'employeur à leur encontre ${ }^{10}$.

Sous l'hypothèse d'une discrimination stratégique, les pénalités salariales, qui ne sont sans doute qu'une partie des discriminations subies, n'empêchent pas certains salariés de devenir délégués syndicaux. Cela ne signifie pas pour autant que cette stratégie discriminatoire échoue. D'autres intérêts que celui de faire carrière motivent leur choix de porter la voix syndicale et peuvent expliquer leur engagement, ce qui ne veut pas dire que ses salariés ne sont pas affectés et affaiblis, au moins sur le long terme, par ces pratiques discriminatoires. La vision du salarié étroitement intéressé qui ne travaille que pour son salaire ou, à l'opposé, celle du syndicaliste héroïque qui se sacrifie pour la cause et que les brimades patronales ne font que renforcer sont des cas extrêmes qu'il convient de nuancer. De nombreux témoignages montrent par exemple que les sacrifices d'abord consentis de bonne grâce par certains syndicalistes finissent par se payer par du découragement et parfois même du renoncement (voir l'expérience relatée par Christian CoROuge dans son dialogue avec Michel PiAlouX dans Résister à la chaîne, 2011). Le recours massif des représentants du personnel au dispositif de rupture conventionnelle, dès qu'il a été mis en place (BOURDIEU, BREDA, 2015), suggère que beaucoup de représentants étaient prêts à quitter leur entreprise en échange d'une indemnité de départ, ce qu'ils ne pouvaient obtenir jusqu'alors du fait du contrôle qu'exerce l'inspection du travail sur les conditions de leur licenciement.

Il est très difficile d'établir si les revendications collectives des délégués syndicaux auraient été finalement moins fortes s'ils avaient été mieux traités. Mais l'assertion inverse, selon laquelle la discrimination des délégués induit des exigences syndicales plus grandes paraît invraisemblable, comme règle générale. Certes, dans certaines circonstances exceptionnelles, il est arrivé que des politiques discriminatoires particulièrement visibles occasionnent des conflits importants et coûteux pour les entreprises, ou qu'elles conduisent à des condamnations juridiques comme on l'a observé de manière fréquente au cours des quinze dernières années. Il n'en demeure pas moins que, dans la plupart des cas, décourager l'action syndicale en réprimant ces leaders est une stratégie qui peut être efficace pour les entreprises.

La question est alors de savoir si la stratégie alternative qui consiste à acheter les délégués syndicaux pour qu'ils acceptent de modérer leurs prétentions ne serait pas plus simple et efficace pour les employeurs. Le surcoût que représentent les quelques avantages, salariaux ou non, consentis pour quelques représentants syndicaux compte sans doute peu en comparaison de ceux que pourraient retirer les employeurs en désamorçant significativement des velléités revendicatrices concernant l'ensemble des salariés.

10. Pour une rationalisation complète de la discrimination stratégique dans un modèle de négociation dynamique, voir BREDA (2011). 
La pratique montre que ce type de stratégie est effectivement mis en œuvre au moins ponctuellement : les cas retentissants de corruption à grande échelle (caisses noires de l'Union des industries et des métiers de la métallurgie [UIMM] pour « fluidifier » le dialogue social, abus notoires de certains comités d'entreprise, etc.) ou encore l'existence de syndicats « jaunes » dans certaines entreprises, sont autant d'exemples de collusions entre les employeurs et les représentants du personnel, au détriment des intérêts des salariés. Dans les faits, ces stratégies de collusion ne sont le plus souvent pas énoncées en tant que telles par ceux qui les mettent en place mais résultent plutôt d'une forme d'accord tacite entre des représentants et des employeurs partageant des intérêts (individuels) bien compris.

Cette stratégie a toutefois des limites. La première est qu'une collusion trop grande serait aussi trop visible et priverait le délégué syndical de la confiance de ses mandants. Tout ce qui pourrait ressembler à du favoritisme est mal perçu par les salariés, même lorsqu'il semble justifié. L'exemple des primes de disponibilité parfois négociées dans les grandes entreprises par certains représentants en raison des sollicitations fréquentes qu'ils reçoivent de la part de leur direction l'illustre bien : certains y voient une juste reconnaissance de l'implication des délégués, tandis que d'autres considèrent qu'il s'agit d'une façon pour l'employeur d'acheter la paix sociale (voir le cas de GRDF décrit par V.-A. CHAPPE et ses co-auteures dans ce numéro, p. 144). La seconde limite tient à la pluralité des syndicats présents dans chaque entreprise : en concurrence les uns avec les autres, ils sont souvent prompts à dénoncer la collusion de l'un d'entre eux avec l'employeur. Il est par conséquent difficile pour ce dernier de mener une telle politique de collusion auprès de l'ensemble des délégués.

Ajoutons que collusion (représentant acheté) ou, au contraire, conflit (représentant discriminé) peuvent être en pratique complémentaires et coexister. La dissuasion que représente la discrimination en cas de comportements trop revendicatifs est d'autant plus crédible que les employeurs en font parfois preuve dans les faits. Elle peut ainsi faciliter l'instauration d'ententes avec les représentants qui redoutent d'être pénalisés. On peut même concevoir que la dénonciation de la corruption non seulement ne suffise pas à l'empêcher mais ne fasse qu'augmenter la division syndicale et, in fine, qu'affaiblir les revendications des salariés.

$\mathrm{Au}$ total, on ne dispose pas ici des moyens de trancher et de dire ce qui pousse une entreprise à pénaliser ses délégués syndicaux plutôt qu'à les acheter. On peut émettre l'hypothèse que l'employeur préférera commencer par essayer d'obtenir la complaisance des représentants par des avantages accordés plus ou moins tacitement, mais qu'en cas d'échec ou face à des syndicalistes particulièrement actifs et peu enclins au compromis, il pourra alors avoir intérêt à les stigmatiser en les discriminant. D'un point de vue empirique, si les pénalités observées reflètent effectivement des comportements stratégiques, nous pouvons conclure qu'en moyenne la pénalisation l'emporte sur les avantages salariaux. L'existence de stratégies aux effets opposés suggère toutefois qu'en mesurant la potentielle discrimination salariale par une pénalité moyenne, on occulte, parfois au sein d'une même entreprise, des cas de primes offertes 
aux représentants qui se montrent conciliants et de pénalités subies encore plus lourdes pour les plus revendicatifs. La distinction entre représentants syndiqués et non syndiqués illustre déjà la diversité potentielle des situations. Et, au sein même du groupe des représentants syndiqués, la pénalité salariale moyenne de l'ordre de $4 \%$ recouvre probablement également une forte hétérogénéité des situations. Cette hétérogénéité est sans doute plus forte que parmi les syndiqués sans mandat, moins susceptibles d'être achetés, puisque n'ayant pas de rôle officiel pouvant influencer le fonctionnement de l'entreprise dans son ensemble. Par conséquent, les pénalités salariales, égales en moyenne, que subissent les syndiqués représentants et non représentants ne sont pas nécessairement synonymes de niveaux de discrimination identiques : ayant davantage d'interactions stratégiques avec les employeurs, les représentants syndiqués peuvent tout à la fois être davantage discriminés et davantage achetés que les autres syndiqués.

D'un point de vue empirique, c'est essentiellement la comparaison des situations des différents types de représentants du personnel, selon leurs attributions officielles, leur syndicat d'appartenance et l'existence de négociations ou de conflits dans leur entreprise qui nous amène à favoriser l'hypothèse d'une discrimination stratégique. Les pénalités salariales encourues par les représentants du personnel sont en effet systématiquement alignées sur les coûts potentiels qu'ils peuvent faire subir à leurs employeurs : plus l'action des représentants est susceptible d'être efficace et d'aboutir à des gains salariaux au détriment des employeurs, moins ils sont payés.

C'est d'abord le contraste de situation entre représentants syndiqués et non syndiqués qui nous conduit à privilégier cette interprétation. Les statistiques sur l'activité des représentants (PAK, PIGNONI, 2014) montrent que les syndiqués sont plus actifs : ils consacrent davantage de temps à leur mandat, passent plus de temps auprès des salariés et plus de temps à les représenter auprès de la hiérarchie. Ils font beaucoup plus souvent des propositions concernant l'activité économique de l'entreprise et appel à des experts ou des structures juridiques ; ils s'appuient fréquemment sur les structures syndicales externes à l'entreprise. Davantage visibles, ils interagissent plus avec les employeurs. Ajoutons que grâce au soutien des structures syndicales, ils sont mieux formés et mieux armés lors des phases formelles de dialogue social. Les intervenants extérieurs dans les entreprises conflictuelles ou dans lesquelles des risques psycho-sociaux ont été identifiés le confirment : les représentants syndiqués connaissent mieux les dossiers et sont moins malléables que leurs homologues non syndiqués.

Mais la distinction la plus importante tient à la fonction qui est en principe dévolue aux délégués syndicaux, à savoir la négociation sur les salaires, l'emploi et les conditions de travail qui doit normalement avoir lieu chaque année avec les employeurs. Contrairement aux autres représentants dont l'intervention peut, au moins sur le papier, davantage contribuer à améliorer le fonctionnement et les performances de leur entreprise, les délégués syndicaux sont surtout cantonnés à participer à un jeu à somme nulle : les gains qu'ils obtiennent se font principalement au détriment des employeurs. Pour le dire autrement, c'est surtout sur les délégués syndicaux que repose la résolution du conflit d'intérêts fondamental entre salariés et actionnaires, 
à savoir comment partager les fruits de la production. Les employeurs peuvent donc avoir intérêt à limiter leur influence par la discrimination parce que ce sont eux, surtout, qui, par la négociation des salaires notamment, peuvent générer une diminution des profits. La comparaison de la situation des délégués syndicaux selon qu'ils ont effectivement négocié les salaires avec leur employeur va dans le sens de cette interprétation : les quelque $30 \%$ de délégués syndicaux qui ne négocient pas avec leur employeur alors qu'ils sont mandatés pour cela ne subissent pas de pénalité salariale, tandis que ceux qui négocient sont fortement pénalisés (entre 10 et $20 \%$ de salaire en moins), d'autant plus que la négociation n'a pas abouti à un accord total avec l'employeur. Les pénalités sont également plus fortes en cas de grève ou de conflit avec l'employeur; elles sont d'autant plus élevées que le conflit a été perdu par les salariés et qu'il a impliqué une part importante des salariés de l'établissement ${ }^{11}$.

Tous ces résultats vont dans le sens de pénalités plus fortes pour les syndicalistes lorsqu'ils ne sont pas en position de force (conflit perdu) et que les coûts qu'ils peuvent occasionner pour leur employeur sont conséquents. Cette hétérogénéité des effets est d'autant plus remarquable qu'il n'a pas été possible de relier par ailleurs les pénalités salariales des délégués syndicaux à des caractéristiques de leur mandat plus directes, comme les heures de délégation, ou la protection contre le licenciement (BREDA, 2014).

Notre théorie d'une discrimination stratégique garde toutefois une zone d'ombre : nous n'avons pas les moyens de déterminer tout à fait si elle relève plutôt d'une discrimination liée à la représentation locale des salariés de l'entreprise, ou d'une discrimination liée à la représentation plus large des intérêts du syndicat. Si les représentants syndiqués ont effectivement pour fonction de négocier et revendiquer au nom des intérêts des salariés de l'entreprise, ils sont également porteurs des intérêts et des revendications plus larges de leur syndicat d'appartenance. Ce que nous montrons, c'est que les pénalités subies par les représentants sont d'autant plus élevées que leur capacité à négocier est importante. En revanche, nous ne pouvons pas affirmer que les intérêts au nom desquels ils négocient sont davantage ceux des salariés de l'entreprise que ceux du syndicat qu'ils représentent.

\section{Autocensure des représentants}

Enfin, une dernière piste d'explication peut être avancée. Il est également envisageable d'expliquer les salaires inférieurs de certains représentants par l'existence possible d'un comportement d'autocensure de leur part : ils renonceraient à mettre en avant leur situation personnelle pour mieux se consacrer à la défense des autres salariés. De nombreux témoignages montrent que les militants perçoivent le sacrifice de carrière comme normal : d'une part, parce qu'ils y sont parfaitement habitués et n'ont jamais réfléchi à la situation sous un angle différent et, d'autre part (peut-être surtout), parce qu'ils sont très attachés à la reconnaissance de leur engagement qu'ils considèrent

11. L'ensemble de ces résultats est détaillé dans BourdieU, BREDA (2014). 
comme incompatible avec une carrière confortable (Beaux, Pialoux, 1999 ; Corouge, PIALOUX, 2011). Les délégués syndicaux les plus investis tiennent ainsi pour suspecte la perspective de promotions ou d'augmentations salariales. Se sacrifier pour leurs collègues représente la manifestation ultime de leur incorruptibilité. À propos d'un accord d'indemnisation, Fred Dijoud, militant à la CFDT (Confédération française démocratique du travail) cité par Libération explique par exemple : «Des copains ont refusé de signer l'accord [de rattrapage salarial pour les syndiqués]. Ils estiment qu'ils avaient conscience des risques de leur engagement syndical. » Un cégétiste affirme par ailleurs qu' « être discriminés, c'est la preuve qu'on n'est pas achetés par la direction $!^{12} »$

Il existe des écarts de salaire de l'ordre de 3 à $4 \%$ en moyenne en défaveur des salariés syndiqués par rapport à leurs collègues non syndiqués de même âge, sexe et niveau d'instruction. Ces pénalités salariales sont plus fortes et peuvent être supérieures à $10 \%$ pour ceux, parmi les syndiqués, qui jouent le rôle de délégué syndical et participent aux négociations avec l'employeur. Les syndiqués ayant un mandat de représentant du personnel autre que délégué syndical subissent des pénalités salariales similaires à celles des syndiqués n'ayant pas de mandat.

Si l'on ne peut prouver avec une certitude totale que ces écarts de salaire reflètent une discrimination, au sens juridique du terme, de nombreux indices le suggèrent. La discrimination salariale représente en outre la partie la plus facilement objectivable (en droit et par le biais de statistiques) d'un ensemble de formes de discrimination et de répressions beaucoup plus larges et bien documentées par les monographies. La discrimination à l'égard des syndicalistes se différencie par ailleurs de la plupart des autres formes de discrimination dans la mesure où elle est la conséquence d'un choix (la participation au syndicat). Elle témoigne sans doute davantage du conflit d'intérêts et du rapport de force entre travail et capital que d'une croyance quant au manque de compétences des syndicalistes ou une pure préférence pour les non syndiqués de la part des employeurs.

Pour rendre le rapport de force entre salariés et employeurs moins défavorable aux premiers dans un contexte de montée des inégalités et de durcissement des conditions de travail, il est nécessaire d'améliorer la capacité des représentants à exprimer et à défendre les intérêts des salariés qu'ils représentent. Pour cela, on pourrait inciter les salariés à être davantage concernés par l'action de leurs représentants, de façon à ce qu'ils puissent les soutenir ou les contester dans les cas manifestes de répression ou de dysfonctionnement. Dans cette perspective, cet article suggère qu'on pourrait commencer par atténuer le lien de subordination entre l'employeur et les représentants des salariés afin de limiter les possibilités de discrimination ou de collusion, et en contrepartie augmenter la dépendance des représentants à l'égard de leurs mandants.

12. Voir Hervé Nathan, « Discrimination syndicale les entreprises se rachètent une conduite », Libération, 2 avril 2002. 


\section{Bibliographie}

Arrow K. J. (1973), “The Theory of Discrimination”, in Ashenfelter O., Rees A. (eds.), Discrimination in Labor Markets, Princeton (N. J.), Princeton University Press, pp. 3-33.

Barnier L.-M., Cochin Y., Debrégeas A., Gélot D., Menghini L., Pignoni M.-T., Reynosa S. (2011), Répression et discrimination syndicales, Paris, Fondation Copernic, Syllepse.

Beaud S., Pialoux M. (1999), Retour sur la condition ouvrière. Enquête aux usines Peugeot de Sochaux-Montbéliard, Paris, Fayard.

BeCKer G. S. (1971), The Economics of Discrimination, Chicago, London, University of Chicago Press.

Bourdieu J., BREDA T. (2015), Les Employeurs face aux représentants du personnel : une situation de discrimination stratégique?, Rapport non publié (écrit dans le cadre d'un projet sur les représentants du personnel financé par la Dares), disponible en ligne : http://www. parisschoolofeconomics.com/breda-thomas/working_papers/Bourdieu_Breda.pdf, consulté le 27 juillet 2016.

BREDA T. (2011), Syndicats, négociations, ou capitalisme familial : effets sur les salaires et la protection de l'emploi, thèse en sciences économiques, École des hautes études en sciences sociales.

BREDA T. (2014), « Les délégués syndicaux sont-ils discriminés ? », Revue économique, vol. 65, $\mathrm{n}^{\mathrm{o}} 6$, pp. 841-880.

CHAPPE V.-A. (2013), « Dénoncer en justice les discriminations syndicales : contribution à une sociologie des appuis conventionnels de l'action judiciaire », Sociologie du travail, vol. 55, $\mathrm{n}^{\mathrm{o}} 3$, pp. 302-321.

CHAPPE V.-A. (2015), « Les discriminations syndicales saisies par le droit à PSA », La Nouvelle Revue du travail [en ligne], ${ }^{\circ} 7$ : https://nrt.revues.org/2324, consulté le 27 juillet 2016.

Chappe V.-A., Denis J.-M., Guillaume C., Pochic S. (2015), Discrimination syndicale et reconnaissance des parcours syndicaux : les deux faces du dialogue social à la française?, Rapport pour la CFDT, Agence d'objectif de l'Ires.

Chappe V.-A., Guillaume C., Pochic S. (2016), « Négocier sur les carrières syndicales pour lutter contre la discrimination. Une appropriation sélective et minimaliste du droit », Travail et Emploi, $\mathrm{n}^{\mathrm{o}} 145$, pp. 121-146.

Coate S., Loury G. C. (1993), "Will Affirmative-Action Policies Eliminate Negative Stereotypes?", The American Economic Review, vol. 83, n 5, pp. 1220-1240.

Corouge C., Pialoux M. (2011), Résister à la chaîne. Dialogue entre un ouvrier de Peugeot et un sociologue, Marseille, Agone.

GiRAUD B. (2013), « Derrière la vitrine du "dialogue social” : les techniques managériales de domestication des conflits du travail », Agone, n ${ }^{\circ} 50$, pp. 33-63.

LOURY G. C. (2002), The Anatomy of Racial Inequality, Cambridge (Mass.), Harvard University Press. 
Lundberg S. J., Startz R. (1983), "Private Discrimination and Social Intervention in Competitive Labor Market", The American Economic Review, vol. 73, n 3 3, pp. 340-347.

PAK M., PignOni M.-T. (2014), «Les représentants du personnel : quelles ressources pour quelles actions ? », Dares Analyses, n 84.

Phelps E. S. (1972), “The Statistical Theory of racism and Sexism”, The American Economic Review, vol. 62, n 4, pp. 659-661.

Semaine sociale Lamy (2004), « Discrimination syndicale : la stratégie de la CGT. Entretien avec François Clerc, chargé des dossiers en discrimination à la CGT », Semaine sociale Lamy, $\mathrm{n}^{\mathrm{o}}$ 1190, pp. 3-10. 


\section{ANNEXE MÉTHODOLOGIQUE}

Nous avons fait deux choix méthodologiques dans le tableau 1. Nous nous sommes d'abord concentrés sur les CDI à temps complet, hors apprentis et stagiaires, hors emplois secondaires, avec heures déclarées annuelles entre 900 et 2 200. L'objectif poursuivi par ces restrictions est d'exclure les salariés précaires et de ce fait moins payés de la comparaison entre les représentants et leurs collègues. De par leur fonction, les représentants ont en effet moins de chances d'être en CDD ou à temps partiel. On s'intéresse ainsi aux écarts de salaire entre les représentants et leurs collègues parmi la main d'œuvre stable (les «insiders »). Lorsque l'on garde, à l'inverse, tous les salariés, y compris ceux qui sont en situation professionnelle plus précaire, on obtient de manière prévisible des écarts légèrement plus faibles (BOURDIEU, BREDA, 2015, tableau A1) ${ }^{13}$.

Notre second choix méthodologique consiste à utiliser les pondérations fournies par la Dares dans les estimations de salaires. L'utilisation de pondérations dans l'analyse économétrique est loin de faire consensus. Pour certains, l'analyse de l'impact causal d'une variable sur une autre ne doit pas se faire en pondérant les observations. Mais notre ambition ici n'est pas de mesurer l'effet causal sur les salaires du fait d'être représentant du personnel; il s'agit simplement de mesurer des écarts de salaire. Selon cette perspective descriptive, il nous semble justifié de pondérer afin d'avoir des estimations les plus représentatives possibles de l'ensemble du marché du travail. $\mathrm{Ne}$ pas les utiliser conduit à trouver des écarts de salaires plutôt plus faibles (BOURDIEU, BREDA, 2015, tableau A2).

\footnotetext{
13. Même s'il se justifie dans le cadre de cette étude, notre choix méthodologique de se concentrer sur les salariés « insiders » peut être critiqué pour les biais de sélection éventuels qu'il induit. Nous avons donc voulu vérifier si les principaux résultats de l'étude sont robustes dès lors que l'on garde dans l'échantillon les salariés à temps partiel et en contrat à durée déterminée (CDD) et que l'on contrôle pour l'effet éventuel de ces variables (en plus des autres contrôles). En général, les pénalités salariales obtenues sur cet échantillon plus large sont :

- légèrement plus faibles pour les salariés syndiqués non-représentants ;

- légèrement plus fortes pour les représentants syndiqués (et les délégués syndicaux) dans les spécifications qui n'incluent pas d'effets fixes par établissement ;

- légèrement plus faibles pour les représentants syndiqués (et les délégués syndicaux) dans les spécifications qui incluent des effets fixes par établissement.

À l'exception de ces légères tendances, les résultats obtenus sont très proches.
} 